\title{
Cordyceps Militaris Alleviates Severity of Murine Acute Lung Injury Through miRNAs-Mediated CXCR2 Inhibition
}

\author{
Sheng Liu Jian Tang Lei Huang Qirong Xu Xiang Ling Jichun Liu \\ Department of Cardiothoracic Surgery, the First Affiliated Hospital of Nanchang University, Nanchang, \\ China
}

\section{Key Words}

Acute lung injury (ALI) • Cordyceps militaris (CM) • miR-1321 • miR-3188 • CXCR2

\begin{abstract}
Background/Aims: Acute lung injury (ALI) and acute respiratory distress syndrome (ARDS) are lethal diseases in humans, and the current treatments have limited therapeutic effects. Cordyceps militaris (CM) is a caterpillar-grown traditional medicinal mushroom, and has been used as a natural invigorant for longevity, endurance, and vitality in China. Recently, purified extracts from $\mathrm{CM}$ have been shown to have beneficial effects on various diseases including cancer. Nevertheless, a role of CM in ALI has not been examined previously. Methods: Here, we used a bleomycin-induced ALI model to study the effects of CM on the severity of ALI in mice. The levels of CXCR2, a receptor for Interleukin 8 (IL-8) in pulmonary microvascular endothelial cells, were examined in different experimental groups. The levels of microRNA (miR)-1321 and miR-3188 were also examined in lung samples and in CM. Adeno-associated viruses carrying miR-1321 and miR-3188 were injected into bleomycin-treated mice for evaluation their effects on the severity of ALI. Results: CM treatment significantly alleviated the severity of bleomycininduced ALI in mice. The increases in lung CXCR2 by bleomycin were significantly reduced by $\mathrm{CM}$ at protein level, but not at mRNA level. CM contained high levels of 2 miRNAs (miR-1321 and miR-3188) that target 3'-UTR of CXCR2 mRNA to inhibit its expression. Overexpression of miR-1321 and miR-3188 in mouse lung through AAV-mediated gene therapy mimicked the effects of CM. Conclusion: CM may alleviate severity of murine ALI through miRNAsmediated CXCR2 inhibition.
\end{abstract}




\section{Introduction}

Acute lung injury (ALI) is a common but complex clinical disease involving acute inflammation, microvascular damage, and increased pulmonary vascular and epithelial permeability, which frequently results in acute respiratory failure into highly lethal acute respiratory distress syndrome (ARDS) [1-4]. ALI and ARDS have been recently reclassified in the Berlin definition [5-7].

Excessive and prolonged activation of neutrophils hallmark ALI, resulting in basement membrane destruction and permeability increases of the alveolar-capillary barrier [812]. Migrating neutrophils also release pro-inflammatory and pro-apoptotic cytokines, e.g. Interleukin 8 (IL-8), to allow the injury progress [8-12]. IL-8 is a potent neutrophil attractant and activator. Previous studies have focused on the formation of anti-IL-8 autoantibody and IL-8 complexes that interact with lung endothelial cells in patients with ARDS [13, 14]. However, IL-8 also triggers cytoskeletal reorganization of microvascular endothelial cells, which is mediated by activation of CXCR2 [15-18]. It has been shown that anti-CXCR2 antibody blocks IL-8-mediated haptotaxis of endothelial cells on collagen, and mediates the increases in vascular permeability in ALI [19].

Cordyceps militaris (CM) is a caterpillar-grown traditional medicinal mushroom, and has been used as a natural invigorant for longevity, endurance, and vitality for thousands of years in China. Some purified extracts from CM has recently shown to have beneficial effects on various diseases, possibly through adjust immunity [20,21]. Recently, effects of CM in inhibiting carcinogenesis in several types of malignant tumors have been reported, and these effects have been proposed to be conducted through reducing cancer metastatic potential and enhancing cytokine induction. Nevertheless, a role of CM in ALI as well as the underlying mechanisms has not been examined previously.

MicroRNA (miRNA) is a class of non-coding small RNA of comprised of around 18-22 nucleotides. MiRNAs regulate the gene expression post-transcriptionally, through its basepairing with the $3^{\prime}$-untranslated region $\left(3^{\prime}\right.$-UTR) of the target mRNA $[22,23]$. Although it has been shown that various miRNAs regulate a great number of biological events, a role of miRNAs in ALI has been ill-defined.

Here, we used a bleomycin-induced ALI model to study the effects of CM on the ALI in mice. The levels of CXCR2 were examined in different experimental groups. The levels of microRNA (miR)-1321 and miR-3188 were also examined in lung samples and in CM. Adeno-associated viruses (AAVs) carrying miR-1321 and miR-3188 were injected into bleomycin-treated mice for evaluation their effects on the severity of ALI. We found that CM treatment significantly alleviated the severity of bleomycin-induced ALI in mice. The increases in CXCR2 in lung by bleomycin were significantly reduced by CM at protein level, but not at mRNA level. CM contained high levels of 2 miRNAs (miR-1321 and miR-3188) that target 3'-UTR of CXCR2 mRNA to inhibit its expression. Overexpression of miR-1321 and miR-3188 in mouse lung via AAV-mediated gene therapy mimicked the effects of CM.

\section{Materials and Methods}

\section{Animal manipulations}

All mouse protocols were approved by the Animal Research and Care Committee at the First Affiliated Hospital of Nanchang University. Female Balb/c mice were purchased from the Laboratory Animal Center of Shanghai Academy of Sciences (Chinese Academy of Sciences, China) and were kept in specific pathogen free (SPF) environment throughout the experiments. Fifteen mice were used in each experimental groups.

The lung injury model was induced by intratracheal instillation of $4 \%$ bleomycin solution (SigmaAldrich, St Louis, MO, USA) at $20 \mathrm{mg} / \mathrm{kg}$ body weight, as has been applied and described before [8]. The control mice received intratracheal saline of same volume. Two weeks after successful induction of ALI, mice received either intratracheal instillation of $0.1 \%$ CM (National Institute for the Control of Pharmaceutical and Biological Products, Beijing, China) or tail vein injection of $10^{9}$ adeno-associated viruses (AAV) carrying 
both miR-1321 and miR-3188. Same titer of AAV carrying null sequence was used as a control for AAVmiRNAs injection. The effects of these treatments on lung function were evaluated after another 4 weeks.

\section{AAV preparation and cell transfections}

Human embryonic kidney 293 cell line (HEK293) was purchased from American Type Culture Collection (ATCC, Rockville, MD, USA), and maintained in Dulbecco's modified Eagle's medium (DMEM, Invitrogen, Carlsbad, CA, USA) supplemented with 10\% fetal bovine serum (FBS; Sigma-Aldrich). HEK293 cells were incubated in a humidified chamber with $5 \% \mathrm{CO}_{2}$ at $37{ }^{\circ} \mathrm{C}$. AAV-miR-1321-miR-3188 and control AAV-null viruses were prepared according to general protocols. Briefly, we used a pAAV-CMV-GFP plasmid (Clontech, Mountain View, CA, USA), a packaging plasmid carrying the serotype 8 rep and cap genes, and a helper plasmid carrying the adenovirus helper functions (Applied Viromics, LLC. Fremont, CA, USA) in this study. The miRNAs were inserted between CMV promoter and GFP reporter by a 2A sequence to allow co-expression of both under the control of the CMV promoter. Sequencing was performed to confirm the correct orientation of the generated plasmids. AAV was prepared by triple transfection of the newly prepared plasmids, R2C8 (containing AAV2 Rep and AAV8 capsid genes) and plAd5 (containing adenovirus helper genes) into HEK293 cells by Lipofectamine 2000 reagent (Invitrogen). The viruses were purified using $\mathrm{CsCl}$ density centrifugation and then titration was performed by a quantitative densitometric dot-blot assay. For cell transduction in vitro, the HEK293 cells were incubated with AAV at a MOI of 100 for 12 hours. For cell transduction in vivo, $10^{9} \mathrm{AAV}$ were injected from the tail vein.

\section{Lung function evaluation}

Mice were anesthetized, after which a small incision was made to expose the trachea, and a cannula was inserted to connect to an inline nebulizer and ventilator. Mice were then challenged with aerosolized PBS followed by increasing doses of methacholine (Sigma-Aldrich). Airway resistance index (RI) and dynamic compliance (Cdyn) were determined by analysis of pressure and flow waveforms.

\section{RNA extraction, reverse transcription and quantitative RT-PCR (RT-qPCR)}

Total RNA and miRNAs were extracted from lung or from cultured cells with miRNeasy mini kit or RNeasy kit (Qiagen, Hilden, Germany), respectively. Complementary DNA (cDNA) was randomly primed from $2 \mu \mathrm{g}$ of total RNA using the Omniscript reverse transcription kit (Qiagen). RT-qPCR was subsequently performed in duplicate with a 1:4 dilution of cDNA using the Quantitect SyBr green PCR system (Qiagen) on a Rotorgene 6000 series PCR machine. All primers were purchased from Qiagen. Data were collected and analyzed with the Rotorgene software accompanying the PCR machine, using 2- $\Delta \mathrm{Ct}$ method for quantification of the relative mRNA expression levels. Values of genes were first normalized against $\alpha$-tubulin, and then compared to controls.

\section{Western blot}

The protein from the mouse lung or cultured cells was extracted using RIPA lysis buffer (1\% NP40, $0.1 \%$ Sodium dodecyl sulfate (SDS), $100 \mu \mathrm{g} / \mathrm{ml}$ phenylmethylsulfonyl fluoride, $0.5 \%$ sodium deoxycholate, in PBS) on ice. Protein concentration was determined using a BCA protein assay kit (Bio-rad, China). Primary antibodies were anti-CXCR2 and anti- $\alpha$-tubulin (all purchased from Cell Signaling, St Louis, MO, USA). $\alpha$-tubulin was used as a protein loading control. Secondary antibody is HRP-conjugated anti-rabbit (Jackson ImmunoResearch Labs, West Grove, PA, USA). Images shown in the figure were representative from 3 repeats. Densitometry of Western blots was quantified with NIH ImageJ software (Bethesda, MA, USA). The protein levels were first normalized to $\alpha$-tubulin, and then normalized to experimental controls.

\section{Immunohistochemistry}

Mouse lung was fixed with 4\% paraformaldehyde for 4 hours, and then cyro-protected in 30\% sucrose overnight. Tissue samples were sectioned in $6 \mu \mathrm{M}$. H\&E staining was performed.

\section{Luciferase-reporter activity assay}

Luciferase-reporters were successfully constructed using molecular cloning technology. Target sequence for CXCR2 miRNA 3'UTR clone was purchased from Creative Biogene (Shirley, NY, USA). HEK293miR-1321, or HEK293-null, or HEK293-as-miR-1321, or HEK293-miR-3188, or HEK293-as-miR-3188 cells 
were seeded in 24-well plates for 24 hours, after which they were transfected with $1 \mu \mathrm{g}$ of Luciferase-reporter plasmids per well using PEI Transfection Reagent. Luciferase activities were measured using the dualluciferase reporter gene assay kit (Promega, Beijing, China), according to the manufacturer's instructions.

\section{Statistical analysis}

All statistical analyses were carried out using the SPSS 18.0 statistical software package. All values are depicted as mean \pm standard deviation and are considered significant if $p<0.05$. All data were statistically analyzed using one-way ANOVA with a Bonferoni correction, followed by a Fisher's exact test to compare two groups.

\section{Results}

\section{CM significantly alleviated the lung injury by bleomycin in mice}

The mouse bleomycin-ALI model has been performed as has been described before [8]. Two weeks after bleomycin treatment, we gave intratracheal instillation of CM to mice, and the control group of mice received intratracheal instillation of saline. After 4 weeks, the mice were examined for lung function. By histology, we found that bleomycin induced significant lung injury in mice, which was alleviated by CM treatment (Fig. 1A). In a cholinergic stimulus (methacholine) test, we detected a dose-dependent increase in lung resistance index (Rl, Fig. 1B) and decrease in Cdyn in response to methacholine (Fig. 1C) in bleomycin-treated mice. Also, the effects of bleomycin on lung function were significantly reduced in bleomycintreated mice that received CM (Fig. 1B-C). These data suggest that CM significantly alleviates the lung injury by bleomycin in mice.

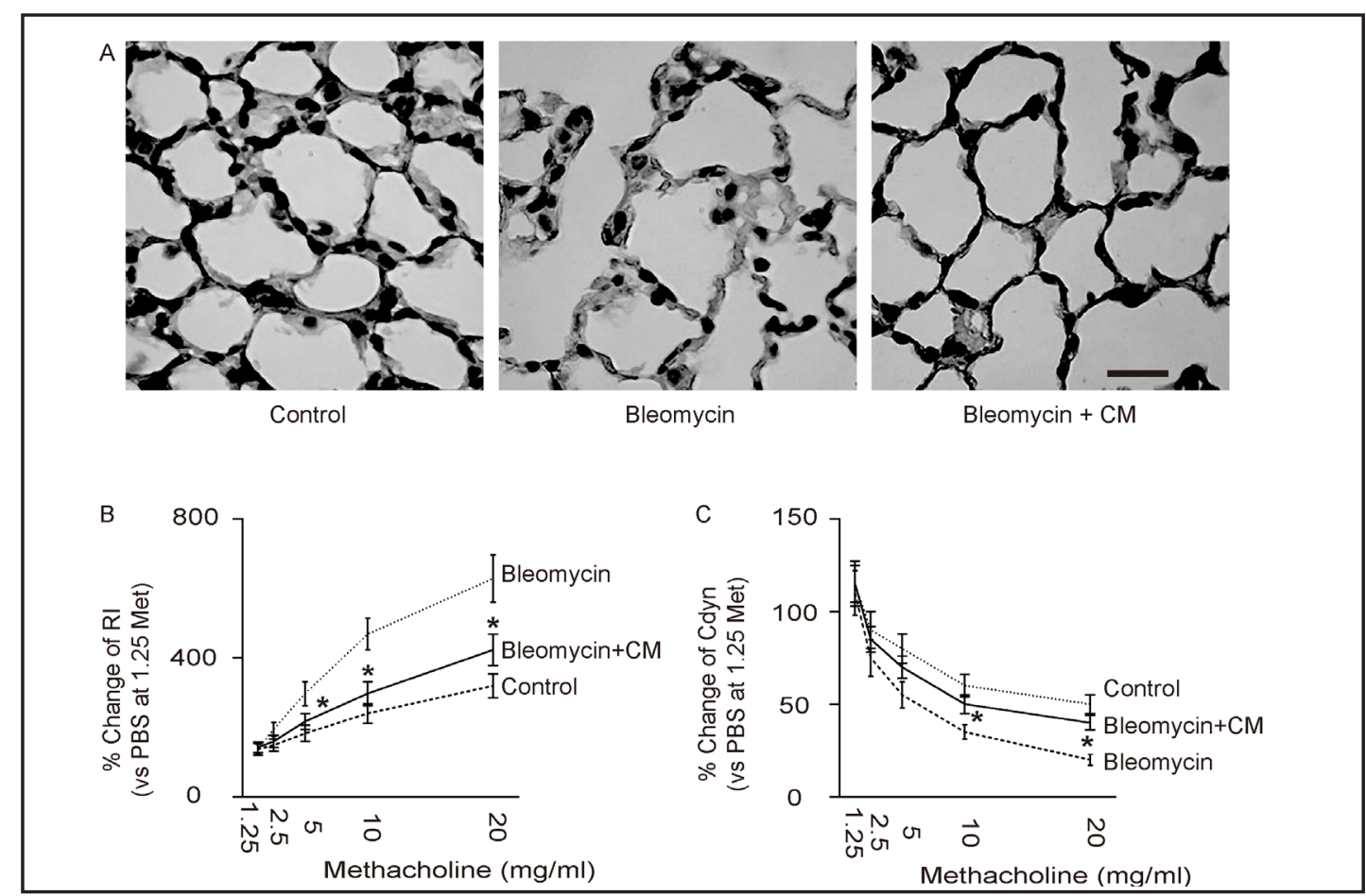

Fig. 1. CM significantly alleviated the lung injury by bleomycin in mice. Two weeks after bleomycin treatment, we gave intratracheal instillation of $\mathrm{CM}$ to mice, and the control group of mice received intratracheal instillation of saline. After 4 weeks, the mice were examined for lung function. (A) Histology. (B-C) Four weeks after CM (six weeks after bleomycin), in a cholinergic stimulus (methacholine) test, we detected a dose-dependent increase in lung resistance index (Rl, B) and decrease in Cdyn in response to methacholine (C) in bleomycin-treated mice. Also, the effects of bleomycin on lung function were significantly reduced in bleomycin-treated mice that received CM (B-C). ${ }^{*} \mathrm{p}<0.05$. N=15. Statistics: one-way ANOVA, followed by a Fish's exact test. Scale bar is $20 \mu \mathrm{m}$. 


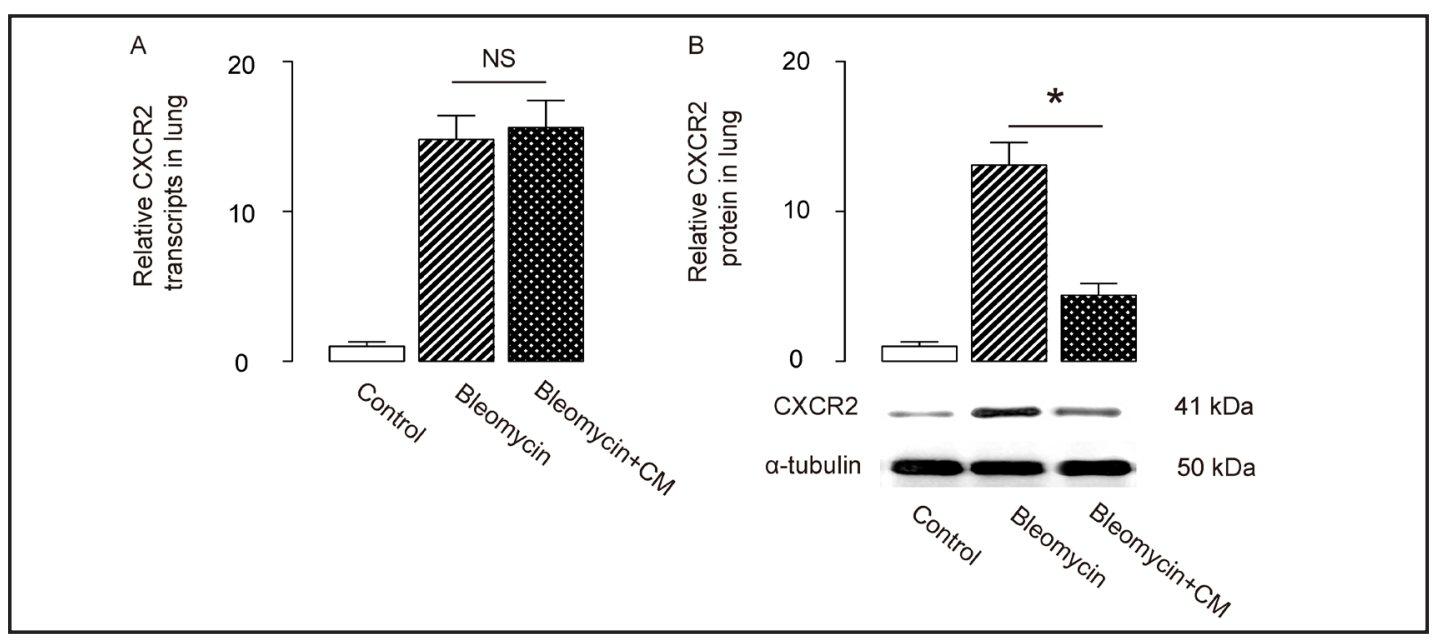

Fig. 2. CM attenuates the bleomycin-induced increases in CXCR2 at protein level but not at mRNA level in mouse lung. CXCR2 levels were analyzed in the mouse lung 4 weeks after CM treatment. (A) At mRNA level, CXCR2 in lung significantly increased by bleomycin treatment, and CM administration did not alter the increases in CXCR2 by bleomycin. (B) At protein level, CXCR2 in lung significantly increased by bleomycin treatment, and CM administration significantly reduced the increases in CXCR2 by bleomycin. ${ }^{*} \mathrm{p}<0.05$. NS: non-significant. N=15. Statistics: one-way ANOVA, followed by a Fish's exact test.

CM attenuates the bleomycin-induced increases in CXCR2 at protein level but not at mRNA level in mouse lung

We then analyzed CXCR2 levels in the mouse lung 4 weeks after CM treatment. At mRNA level, CXCR2 in lung significantly increased by bleomycin treatment, and CM administration did not alter the increases in CXCR2 by bleomycin (Fig. 2A). However, at protein level, CXCR2 in lung significantly increased by bleomycin treatment, and CM administration significantly reduced the increases in CXCR2 by bleomycin (Fig. 2B). These data not only suggest that CXCR2 protein but not mRNA, was regulated by CM in bleomycin-treated mouse lung, but also suggest that the alteration of CXCR2 levels by CM may result from translational control, rather than from promoter activation at transcription level.

MiR-1321 and miR-3188 are two components in CM and target 3'-UTR of CXCR2 mRNA to inhibit its translation in bleomycin-treated mouse lung

Since we found that the increases in CXCR2 protein, but not mRNA, significantly reduced after CM administration in bleomycin-treated mouse lung, we hypothesize that CXCR2 in the bleomycin-treated mouse lung may be regulated by miRNAs from CM. Thus, we performed bioinformatics analysis of CXCR2 target sequence. From all binding miRNAs for 3'UTR of CXCR2, we found that two miRNAs [miR-1321: binding sites in the CXCR2 mRNA sequence 3'UTR ranged from 33th base site to 39th base site (Fig. 3A); miR-3188: binding sites in the CXCR2 mRNA sequence 3'UTR ranged from 60th base site to 67th base site (Fig. 3B)] significantly increased in $\mathrm{CM}$ and bleomycin -treated mouse lung, but did not increased by bleomycin-treatment alone (Fig. 3C). Moreover, in CM, these two miRNAs were detected highly expressed (Fig. 3C). HEK293-miR-1321, HEK293-null (as a control) and HEK293-antisense (as)-miR-1321 cells were prepared by transfection and confirmed of miR-1321 modification (Fig. 3D). These cells were then transfected with $1 \mu \mathrm{g}$ of CXCR2-3'UTR luciferase-reporter plasmid. The luciferase activities in these cells suggest that miR-1321 targets 3'UTR of CXCR2 mRNA to inhibit its expression (Fig. 3E). Similarly, HEK293-miR-3188, HEK293-null (as a control) and HEK293-as-miR-3188 cells were prepared by transfection and confirmed of miR-3188 modification (Fig. 3F). These cells were then transfected with $1 \mu \mathrm{g}$ of CXCR23'UTR luciferase-reporter plasmid. The luciferase activities in these cells suggest that miR3188 also targets 3'UTR of CXCR2 mRNA to inhibit its expression (Fig. 3G). Together, these 
Fig. 3. $M i R-1321$ and miR-3188 are two components in $\mathrm{CM}$ and target 3'-UTR of CXCR2 mRNA to inhibit its translation. (AB) Bioinformatics analysis of CXCR2 target sequence. From all binding miRNAs for 3'UTR of CXCR2, we found that two miRNAs [miR-1321: binding sites in the CXCR2 mRNA sequence 3'UTR ranged from 33th base site to 39th base site (A); miR-3188: binding sites in the CXCR2 mRNA sequence 3'UTR ranged from 60th base site to 67 th base site (B)]. (C) Levels of miR-1321 and miR-3188 in mouse lung and in CM. (D-E) HEK293$\mathrm{m}$ i R - 1321 , HEK293-null (as a control) and HEK293-antisense (as)-miR-1321 cells were prepared by transfection and confirmed of miR-1321 modification (D). These cells were then transfected with

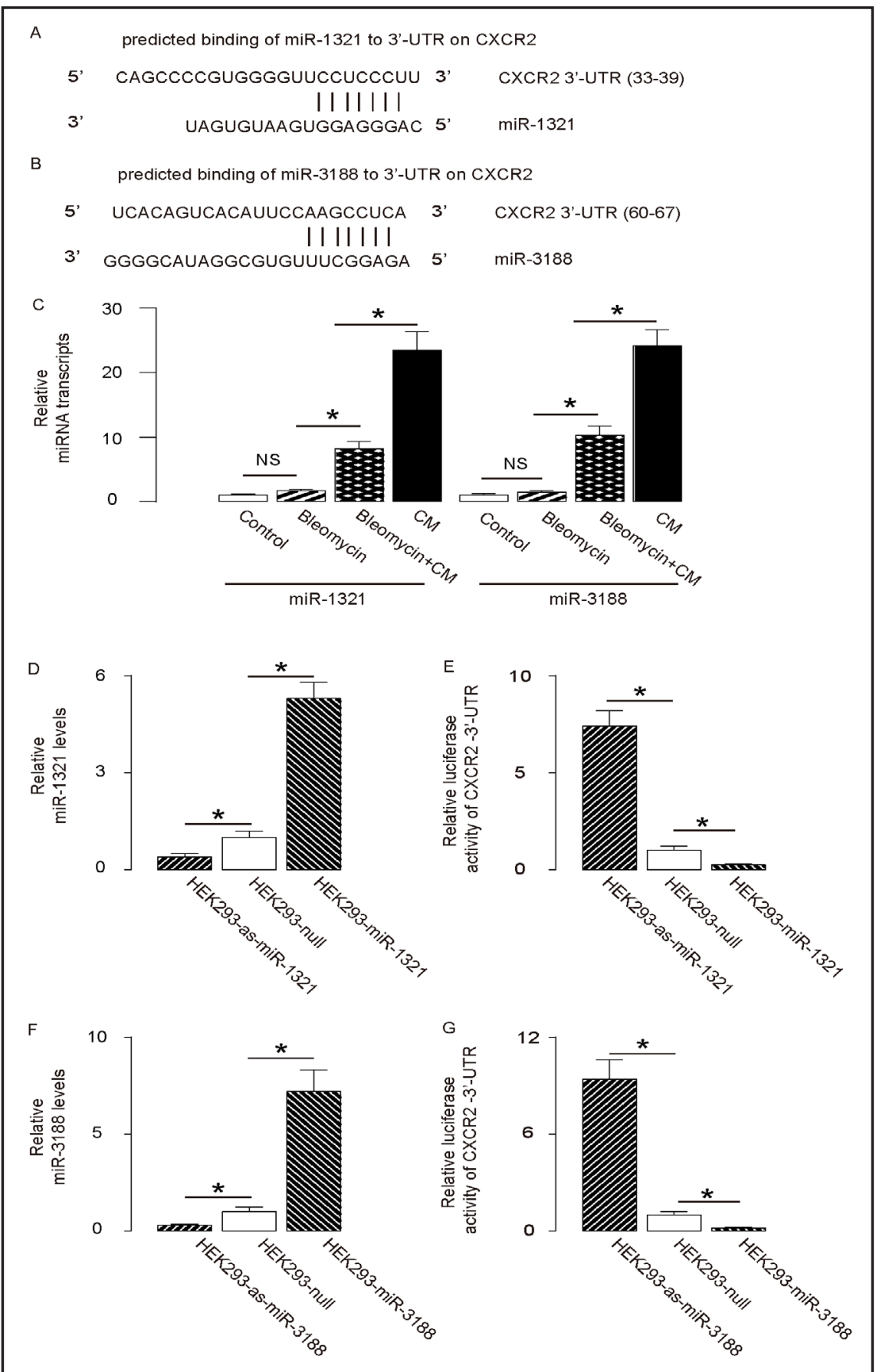

$1 \mu \mathrm{g}$ of CXCR2-3'UTR luciferase-reporter plasmid. The luciferase activities in these cells suggest that miR1321 targets 3'UTR of CXCR2 mRNA to inhibit its expression (E). (F-G) HEK293-miR-3188, HEK293-null (as a control) and HEK293-as-miR-3188 cells were prepared by transfection and confirmed of miR-3188 modification (F). These cells were then transfected with $1 \mu \mathrm{g}$ of CXCR2-3'UTR luciferase-reporter plasmid. The luciferase activities in these cells suggest that miR-3188 also targets 3'UTR of CXCR2 mRNA to inhibit its expression $(\mathrm{G}) .{ }^{*} \mathrm{p}<0.05 . \mathrm{N}=15$. Statistics: one-way ANOVA, followed by a Fish's exact test.

data suggest that miR-1321 and miR-3188 are two components in CM and target 3'-UTR of CXCR2 mRNA to inhibit its translation in bleomycin-treated mouse lung. 
Fig. 4. Combined treatments with miR1321 and miR-3188 mimicked the effects of CM in bleomycin-treated mouse. (A-B) Two weeks after bleomycin treatment, mice received AAVs carrying both miR1321 and miR-3188 for 4 weeks, while control mice received null AAVs. After another 4 weeks, we detected a significantly reduction in RI (A) and a significantly increase in Cdyn (B) in response to a high dose methacholine in AAV-miRNAs-treated mice, compared to the mice that received bleomycin and control viruses. ${ }^{*} \mathrm{p}<0.05$. $\mathrm{N}=15$. Statistics: one-way ANOVA, followed by a Fish's exact test.

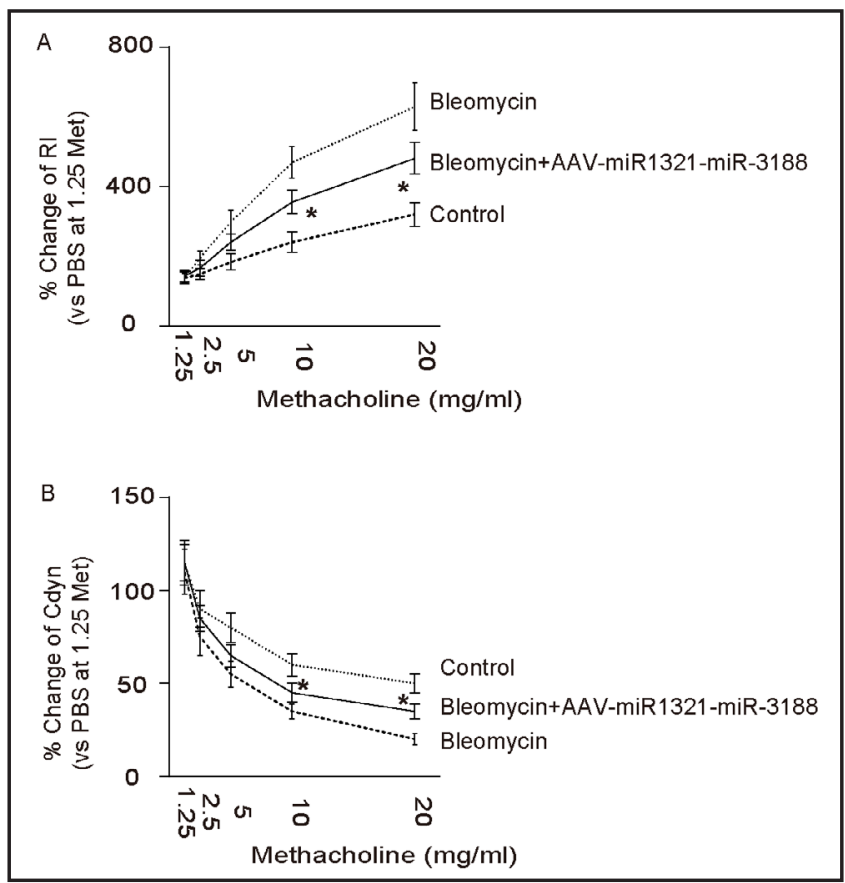

Combined treatments with miR-1321 and miR-3188 mimicked the effects of CM in bleomycin-treated mouse

In order to figure out whether miR-1321 and miR-3188 are critical for the protective effects of CM against bleomycin-induced lung injury, we prepared an AAV carrying both miRNAs. Two weeks after bleomycin treatment, mice received these AAVs for 4 weeks, while control mice received null AAVs. After another 4 weeks, we detected a significantly reduction in RI and a significantly increase in Cdyn in response to

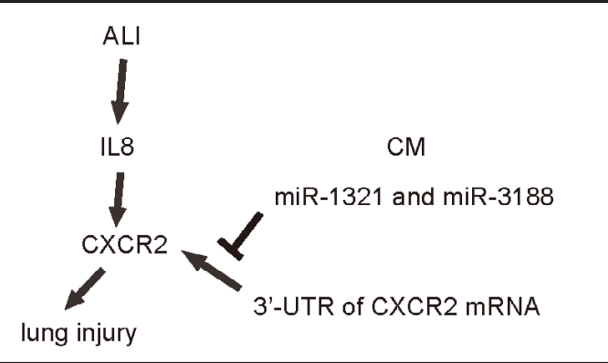

Fig. 5. Schematic of the model. CM protects the mouse lung from acute injury through miR-1321 and miR-3188-mediated suppression of CXCR2, the receptor for IL-8 signaling. a high dose methacholine in AAV-miRNAs-treated mice, compared to the mice that received bleomycin and control viruses (Fig. 4A-B). These data suggest that combined treatments with miR-1321 and miR-3188 mimicks the effects of CM in bleomycin-treated mouse. Taken together, our study suggests a role of CM in protection of lung injury through miR-1321 and miR-3188-mediated suppression of CXCR2, the receptor for IL-8 signaling (Fig. 5).

\section{Discussion}

Effective treatments of ALI and ARDS are highly needed to reduce the lethality of these common diseases [1-4]. Since ALI is characterize by excessive and prolonged activation of neutrophils, which results in basement membrane destruction and permeability increases of the alveolar-capillary barrier [8-12], previous efforts have been focusing on the modulation of the immunity. Specifically, IL-8 is a critical cytokine that is produced and released by neutrophils. IL-8 targets microvascular endothelial cells via its receptor CXCR1 and CXCR2 to trigger the changes in permeability of vascular endothelium to facilitate the lung injury [812]. Hence, it may be logic that inhibition of CXCR2 in lung endothelial cells may be beneficial. Modulation of CXCR2 levels may be through promoter activity, translation control and 
post-translational modification of the protein, etc. MiRNAs are critical for regulation of mRNA translation for many proteins $[22,23]$. However, previous studies have not provided strong evidence of miRNA on CXCR2 in ALI. Here, we show solid evidence that CM contained two component miRNAs that target 3'-UTR of CXCR2 to suppress its translation. CM has been used as a natural invigorant for longevity, endurance, and vitality for thousands of years in China. More and more components in CM have been identified to have thearapeutic effects on various diseases, including cancer [20, 21, 24, 25]. For example, Cordycepin ( 3 '-deoxyadenosine) is a nucleoside derivative that has been purified from CM. Multiple biological activities of Cordycepin have been shown to include antiviral, antifungal, antiinflammatory, antihyperglycemic, and antiatherosclerotic activities. Specifically, Cordycepin has been reported recently to attenuate the inflammatory process in the Ovadriven asthmatic mice [26]. Since the asthma and ALI share some pathological processes, we think that the anti-ALI effects of CM may partially come from miR-3188 and miR-1321, and partially from Cordycepin. Future experiments may be applied to clarify the exact contribution of these components in $\mathrm{CM}$ to the protective effects against lung injury.

Moreover, the secondary molecular effects of CXCR2 suppression may be analyzed. For example, how are the changes in angiogenic factors, e.g. vascular endothelial growth factor (VEGF) family members [27-29] in the mouse lung by CXCR2 suppression? Furthermore, do miR-3188 and miR-1321 have other targets that may affect the pathogenesis in ALI? To address these questions, we may be able to significantly improve our understanding of the therapeutic effects of CM on ALI.

Our data clearly demonstrate independent inhibitory roles of miR-1321 and miR3188 in the translational control of CXCR2. From the degree of inhibition, it seems that the inhibitory potent of both miRNAs is similar, which is not surprising, since both miRNAs bind to the close region on the 3'-UTR of CXCR2 mRNA, and have exactly same base pairing. The two miRNAs may reinforce the effects of each other to strengthen the suppression.

In summary, in the current study, we report a previously unappreciated protective effect of CM against lung injury. Moreover, we propose that the protection may largely come from the two miRNA components of CM.

\section{Disclosure Statement}

The authors have declared that no conflict of interest exists.

\section{Reference}

1 Ashbaugh DG, Bigelow DB, Petty TL, Levine BE: Acute respiratory distress in adults. Lancet 1967;2:319323.

$\checkmark 2$ Herold S, Gabrielli NM, Vadasz I: Novel concepts of acute lung injury and alveolar-capillary barrier dysfunction. Am J Physiol Lung Cell Mol Physiol 2013;305:L665-681.

-3 Dengler V, Downey GP, Tuder RM, Eltzschig HK, Schmidt EP: Neutrophil intercellular communication in acute lung injury. Emerging roles of microparticles and gap junctions. Am J Respir Cell Mol Biol 2013;49:15.

4 Vlaar AP, Juffermans NP: Transfusion-related acute lung injury: A clinical review. Lancet 2013;382:984994.

5 Kangelaris KN, Calfee CS, May AK, Zhuo H, Matthay MA, Ware LB: Is there still a role for the lung injury score in the era of the berlin definition ards? Ann intensive care 2014;4:4.

6 Khemani RG, Wilson DF, Esteban A, Ferguson ND: Evaluating the berlin definition in pediatric ards. Intensive Care Med 2013;39:2213-2216. 


\section{Cellular Physiology Cell Physiol Biochem 2015;36:2003-2011

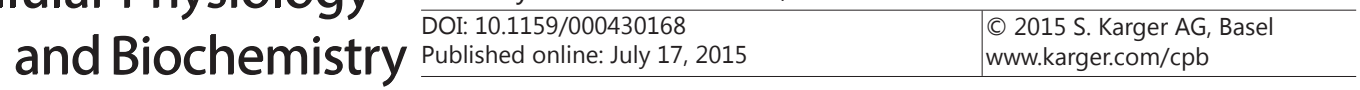

Liu et al.: Cordyceps Militaris Alleviates ALI Through CXCR2

7 Thompson BT, Matthay MA: The berlin definition of ards versus pathological evidence of diffuse alveolar damage. Am J Respir Crit Care Med 2013;187:675-677.

8 Ji Y, Gao F, Sun B, Hao J, Liu Z: Angiotensin-converting enzyme 2 inhibits apoptosis of pulmonary endothelial cells during acute lung injury through suppressing smad2 phosphorylation. Cell Physiol Biochem 2015;35:2203-2212.

-9 Yin X, Liang Z, Yun Y, Pei L: Intravenous transplantation of bmp2-transduced endothelial progenitor cells attenuates lipopolysaccharide-induced acute lung injury in rats. Cell Physiol Biochem 2015;35:2149-2158.

10 Uhlig S, Yang Y, Waade J, Wittenberg C, Babendreyer A, Kuebler WM: Differential regulation of lung endothelial permeability in vitro and in situ. Cell Physiol Biochem 2014;34:1-19.

11 Landgraf MA, Silva RC, Correa-Costa M, Hiyane MI, Carvalho MH, Landgraf RG, Camara NO: Leptin downregulates lps-induced lung injury: Role of corticosterone and insulin. Cell Physiol Biochem 2014;33:835-846.

12 Cai DS, Zhou H, Liu WW, Pei L: Protective effects of bone marrow-derived endothelial progenitor cells and houttuynia cordata in lipopolysaccharide-induced acute lung injury in rats. Cell Physiol Biochem 2013;32:1577-1586.

13 Allen TC, Kurdowska A: Interleukin 8 and acute lung injury. Arch Pathol Lab Med 2014;138:266-269.

14 Silva RC, Landgraf MA, Correa-Costa M, Semedo P, Cenedeze MA, Pacheco-Silva A, Landgraf RG, Camara NO: Acute kidney injury reduces phagocytic and microbicidal capacities of alveolar macrophages. Cell Physiol Biochem 2013;31:179-188.

15 Konrad FM, Reutershan J: Cxcr2 in acute lung injury. Mediators Inflamm 2012;2012:740987.

16 Zarbock A, Allegretti M, Ley K: Therapeutic inhibition of cxcr2 by reparixin attenuates acute lung injury in mice. Br J Pharmacol 2008;155:357-364.

17 Strieter RM, Keane MP, Burdick MD, Sakkour A, Murray LA, Belperio JA: The role of cxcr2/cxcr2 ligands in acute lung injury. Curr Drug Targets Inflamm Allergy 2005;4:299-303.

18 Lomas-Neira JL, Chung CS, Grutkoski PS, Miller EJ, Ayala A: Cxcr2 inhibition suppresses hemorrhageinduced priming for acute lung injury in mice. J Leukoc Biol 2004;76:58-64.

19 Belperio JA, Keane MP, Burdick MD, Londhe V, Xue YY, Li K, Phillips RJ, Strieter RM: Critical role for cxcr2 and cxcr2 ligands during the pathogenesis of ventilator-induced lung injury. J Clin Invest 2002;110:17031716.

20 Zhu SJ, Pan J, Zhao B, Liang J, Ze-Yu W, Yang JJ: Comparisons on enhancing the immunity of fresh and dry cordyceps militaris in vivo and in vitro. J Ethnopharmacol 2013;149:713-719.

21 Wang M, Meng XY, Yang RL, Qin T, Wang XY, Zhang KY, Fei CZ, Li Y, Hu Y, Xue FQ: Cordyceps militaris polysaccharides can enhance the immunity and antioxidation activity in immunosuppressed mice. Carbohydr Polym 2012;89:461-466.

22 Di Leva G, Croce CM: Mirna profiling of cancer. Curr Opin Genet Dev 2013;23:3-11.

23 Pereira DM, Rodrigues PM, Borralho PM, Rodrigues CM: Delivering the promise of mirna cancer therapeutics. Drug Discov Today 2013;18:282-289.

24 Jeong MH, Lee CM, Lee SW, Seo SY, Seo MJ, Kang BW, Jeong YK, Choi YJ, Yang KM, Jo WS: Cordycepinenriched cordyceps militaris induces immunomodulation and tumor growth delay in mouse-derived breast cancer. Oncol Rep 2013;30:1996-2002.

25 Mollah ML, Park DK, Park HJ: Cordyceps militaris grown on germinated soybean induces g2/m cell cycle arrest through downregulation of cyclin b1 and cdc25c in human colon cancer ht-29 cells. Evid Based Complement Alternat Med 2012;2012:249217.

-26 Yang X, Li Y, He Y, Li T, Wang W, Zhang J, Wei J, Deng Y, Lin R: Cordycepin alleviates airway hyperreactivity in a murine model of asthma by attenuating the inflammatory process. Int Immunopharmacol 2015;26:401-408.

27 Xiao X, Guo P, Chen Z, El-Gohary Y, Wiersch J, Gaffar I, Prasadan K, Shiota C, Gittes GK: Hypoglycemia reduces vascular endothelial growth factor a production by pancreatic beta cells as a regulator of beta cell mass. J Biol Chem 2013;288:8636-8646.

28 Cao Y: Angiogenesis and vascular functions in modulation of obesity, adipose metabolism, and insulin sensitivity. Cell Metab 2013;18:478-489.

29 Murukesh N, Dive C, Jayson GC: Biomarkers of angiogenesis and their role in the development of vegf inhibitors. Br J Cancer 2010;102:8-18. 